
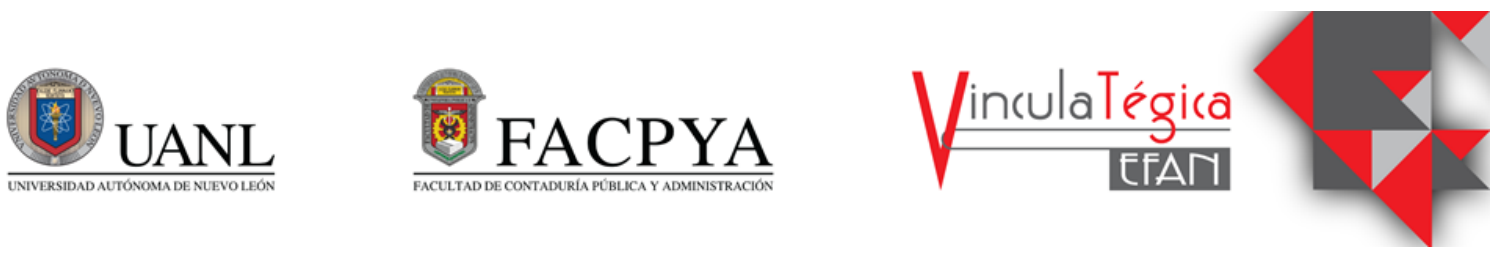

\title{
Análisis dicotómico en las definiciones del concepto de trabajo significativo
}

\author{
Amparo Guadalupe Espinosa Domínguez ${ }^{1}$, Arístides Alfredo Vara-Horna ${ }^{2}$ y María Elvira \\ Cantú González ${ }^{3}$ \\ ${ }^{1}$ Universidad Autónoma de Nuevo León, lupita_espinosa@prodigy.net.mx, Av.Universidad s/n Cd. \\ Universitaria, San Nicolás de los Garza, N.L.Cel.811.58.55.612 \\ ${ }^{2}$ Universidad de San Martín de Porres, aristidesvara@gmail.com, Av. Brasil 1857 - Jesus Maria, Lima, \\ Peru,811.58.55.612 \\ ${ }^{3}$ Universidad Autónoma de Nuevo León, elviracantugonzalez@yahoo.com.mx, Av. Universidad s/n Cd. \\ Universitaria, San Nicolás de los Garza, N.L.Cel.818.02.94.532
}

Información del artículo revisado por pares

Fecha de aceptación: junio-2021

Fecha de publicación en línea: diciembre-2021

DOI: https://doi.org/10.29105/vtga7.2-40

\section{Resumen}

La pandemia derivada del COVID-19, ha perturbado las dinámicas sociales y organizacionales. El propósito de este estudio es analizar el concepto de trabajo significativo desde la dicotomía de las perspectivas de diferentes autores. Se analizaron 21 artículos de revistas internacionales y se hicieron clasificaciones de acuerdo con elementos que mostraban variadas experiencias: intrínseca, extrínseca, positiva y negativa, además de la dimensión de continuidad en el tiempo. Los resultados confirman dicotomía e incluso antagonismo en las definiciones estudiadas. Se concluye la importancia de promover homogeneidad en la definición del trabajo significativo para un correcto estudio del concepto. Además, se sugiere un estudio profundo de la dinámica en la generación de trabajo significativo, de acuerdo con los escenarios en que el individuo se desenvuelve social y organizacionalmente.

Palabras clave: Dicotomía, Experiencia extrínseca, Keywords: Dichotomy, extrinsic experience, Experiencia intrínseca, Trabajo significativo.

\begin{abstract}
The COVID-19 pandemic has disturbed social and organizational dynamics. The purpose of this study is to analyze the concept of meaningful work from different authors' perspectives. For purposes of this analysis twenty-one articles from different international journals were analyzed and classifications were made according to elements that showed varied experiences: intrinsic, extrinsic, positive and negative, in addition to the dimension of continuity over time. The results showed dichotomy and even antagonism in the studied definitions. In conclusion, it is important to promote with consistency the definition of meaningful work for a correct study of the concept. Additionally, it is suggested a deep study of the dynamics in the creation of meaningful work, according to the scenarios in which the individual develops socially and organizationally.
\end{abstract}




\section{INTRODUCCIÓN}

A nivel internacional el trabajo se ve profundamente afectado por la pandemia originada por el COVID-19, las perturbaciones que se presentan no solo son económicas y sociales, sino que pone en peligro la forma y sentido de la vida a corto y largo plazo, tanto de las organizaciones como de los individuos dentro de ellas.

Este estudio muestra un análisis descriptivo de las definiciones del concepto de trabajo significativo y su dicotomía en tiempos de COVID-19.

Al analizar el concepto de trabajo significativo desde la raíz etimológica de cada uno de sus componentes, podría crear controversia al ser la palabra trabajo un derivado de yugo, mientras que "significado" se percibe como una idea que genera bienestar.

Por lo anterior y dadas las definiciones publicadas del concepto de Trabajo Significativo o Meaningful Work, a lo largo de casi 40 años de investigación; surge el interés de analizar las definiciones del concepto desde la dicotomía identificada, con respecto a si, el trabajo significativo es una experiencia interna o tiene que ver con el exterior y el posible antagonismo de lo positivo o negativo que puede llegar a ser el trabajo para el individuo en las organizaciones.

Por otro lado, pero en el mismo sentido, surge la pregunta ¿las definiciones de trabajo significativo que existen muestran incongruencia? ¿En tiempos de COVID-19, es relevante la significancia del trabajo?

En este estudio se analizan definiciones y se identifican elementos que pueden causar confusión y por lo tanto limitar la comprensión de lo que es el trabajo significativo.

\section{MARCO TEÓRICO}

El trabajo significativo ha sido poco estudiado en México, sin embargo, en otros países han avanzado en la investigación del concepto.

Según Stephan (2020) el trabajo significativo se refiere a la experiencia personal del trabajo como enriquecedor y útil, además de ser una fuente de motivación intrínseca enfocada a un bien mayor con propósito y significado. Por el contrario, para Bailey \& Madden (2017), el trabajo significativo se vive tanto en el ambiente laboral como el personal y puede ser una experiencia profunda, conmovedora e incluso incómoda.

Por la polaridad en las definiciones del concepto, es que se considera analizar la dicotomía en cuanto a si es una experiencia interna o externa. Y por otro lado, en menor medida y de acuerdo con los datos encontrados, el antagonismo de las definiciones en cuanto a lo positivo o negativo de la experiencia.

Sobre la base de las consideraciones anteriores se encuentran definiciones del concepto de trabajo significativo y una brecha de conocimiento en cuanto al análisis de la dicotomía y antagonismo de éstas. A continuación, las definiciones de acuerdo con la perspectiva de cada autor.

\subsection{Definiciones.}

Las perspectivas teóricas y los estudios empíricos en torno a las definiciones de trabajo significativo despiertan interés por su estudio. Sobre todo, al identificar polaridades en las definiciones propuestas por diferentes autores y en contribución a esclarecer la 
percepción del concepto.

\subsubsection{Trabajo Significativo.}

Como se menciona en la introducción, la definición del concepto "trabajo" desde su raíz etimológica: proviene de trabajar y ésta del latín tripaliare. "Tripaliare viene de tripalium (tres palos). Tripalium era un yugo hecho con tres (tri) palos (palus) en los cuales amarraban a los esclavos para azotarlos" (RAE), por lo que se deduce que el concepto tiene una conceptualización nada disfrutable.

Por otro lado, la raíz etimológica de "significado" está formada con raíces latinas y significa: objeto, acción, relación, idea o emoción que se transmite de una persona a otra. Sus componentes léxicos son: signa (señal, marca), facere (hacer), más sufijo ado (que recibe acción). (Diccionario Etimológico Castellano en Línea 2002-2020)

Cómo se observa el concepto en general y etimológicamente, se puede percibir como una actividad negativa o por lo menos no disfrutable.

Sin embargo, el constructo "significación" tiene un valor positivo en la literatura y en conjunto ambas palabras: "trabajo significativo", tema de este estudio, es un trabajo que se experimenta como particularmente significativo y con significado positivo para las personas y su entorno (Hackman y Oldham, 1976; Rosso, Dekas y Wrzeniewski, 2010; Allan y Liu, 2020;).

Para Bailey \& Madden (2017), el trabajo significativo es una experiencia que va más allá del lugar de trabajo y llega a los ámbitos de la vida personal del individuo, al grado que se convierte en una experiencia profunda, conmovedora y que incluso puede llegar a que el individuo sienta incomodidad.

En esté estudio se encuentran definiciones que se han manejado en las investigaciones de trabajo significativo, en el período de 1976 y a 2020. Se identifican elementos dicotómicos que los autores han aportado al conocimiento con respecto al trabajo significativo.

Para Schabram, K., \& Maitlis, S. (2017), la vocación tiene que ver con el trabajo significativo. En relación a que puede resultar una experiencia intensa. Esto se debe a que la vocación responde a la importancia que el trabajo tiene para las personas y que está ligado a sus valores. Por lo que puede tener efectos negativos en el logro de un equilibrio entre el trabajo y la vida personal; volviendose un sacrificio personal, si el empleado superpone el trabajo significativo sobre su tiempo libre y familiar.

El trabajo es concebido por los individuos como una fuente de ingresos, que sirve únicamente para satisfacer necesidades económicas, para otros es una forma de relacionarse y hacer amigos, o bien, como una forma de mantenerse ocupados. Incluso, hay quienes ven el trabajo como una forma de estatus social o por el contrario, hay quienes ven el trabajo como una fuente de constante preocupación e inseguridad. Brief, A. P., \& Nord, W. R. (Eds.). (1990).

Desde la identificación del individuo con su trabajo, el trabajo significativo tiene un propósito de vida más alla del yo, lo que esta determinado por los valores, creencias, aspiraciones o metas que constituyen la identidad, (Burke \& Stets, 2009).

Por su parte Ciulla (2000), argumenta que el significado es interno y subjetivo, dado que los individuos difieren de lo que encuentran significativo. Sin embargo, también considera 
que es externo y objetivo, ya que lo que sucede en el mundo da forma y contribuye a lo que hacemos y podemos no encontrar significativo. Incluso, en cualquier tipo de actividad que se realice como parte de un empleo remunerado o de tiempo libre del individuo.

Otra forma de describir el trabajo significativo es desde tres orientaciones: el empleo como fuente de bienestar, la carrera como fuente de avance y prestigio y estatus o como una vocación que contribuye a un bien mayor, Wrzeniewski, (2001).

Chalofsky, N. (2003), enfoca el concepto de trabajo significativo desde la esencia de, lo que el individuo hace y que le genera una sensación de plenitud a su vida, por lo que el trabajo significativo puede contribuir de manera importante a alcanzar el propósito de vida del individuo.

\subsubsection{Experiencia intrínseca.}

De acuerdo con Korotkov, D.L. (1998) el significado tiene que ver con el grado en que la vida tiene sentido emocional para el individuo y que las situaciones que enfrenta las percibe como un valor de inversión y compromiso energético. Queda de manifiesto entonces, que se refiere a los aspectos del individuo que tienen que ver con el valor que deposita en su trabajo de acuerdo a sus creencias o filosofía de vida, elementos totalmente intrínsecos al individuo.

Por su parte Ciulla (2005), confirma que el significado es interno y subjetivo, dado que el individuo logra encontrar y dar diferentes significados al trabajo.

La significación en el trabajo se relaciona con un sentido de valor inherente a la vida y de vivir una vida, que valga la pena vivir. En un sentido más amplio es una evaluación general del trabajo en cuanto a si es intrínsecamente valioso y qué, además, vale la pena realizarlo, Martela, \& Steger (2016).

En el marco de las observaciones anteriores, se identifican argumentos a favor de la experiencia intrínseca del trabajo significativo.

\subsubsection{Experiencia extrínseca.}

En cuanto a la relación que el individuo tiene con su entorno y del cual obtiene estímulos para encontrar significancia en el trabajo, se describen tres orientaciones en la afectación del trabajo significativo. El empelo, como fuente de bienestar, la carrera como fuente de prestigio y desarrollo y la vocación o llamado que tiene que ver con el esfuerzo que el individuo esta dispuesto hacer para sobresalir Wrzesniewski \& Dutton (2001).

Realizar un trabajo significativo se considera una necesidad económica básica y fundamental, Yeoman, R. (2014). Por otro lado pero en el mismo sentido, el significado existencial de la significación tiene espacio entre lo ideal y la realidad, lo que lleva al individuo a buscar unidad con los demás, a servir y a expresarse activamente, MarjoleinLips-Wiersma Lani (2009), encontrado sentido en elementos externos a el mismo y buscando impactar en un bien mayor.

Con lo anterior se confirma que para que se genere la experiencia extrínseca del trabajo significativo el individuo tendrá que buscar estímulos fuera de sí, que lo incentiven a lograr sentido y significado en su labor dentro de la organización. 


\subsubsection{Experiencia positiva.}

El trabajo significativo es un componente fundamental en el bienestar del individuo, dada la importancia que éste cree, que su trabajo tiene. La experiencia de vivir el trabajo significativo generalmente promueve resultados positivos y en beneficio para las personas y las organizaciones, Rosso, Dekas, and Wrzeniewski, (2010).

La significación en el trabajo es una experiencia enriquecedora y útil para el individuo, además de ser una forma de expresarse a sí mismo y una fuente de motivación intrínseca que conduce a un mayor sentido de propósito y significado. Stephan, Tavares, Carvalho, Ramalho, Santos, \& van Veldhoven (2020).

Por su parte, Allan \& Liu (2020), descroben al trabajo significativo como una experiencia intrísecamente valiosa y como un factor crítico que lleva a generar resultados positivos en la organización, además de promover el compromiso, la satisfacción laboral y el desempeño.

Finalmente y respecto al la experiencia positiva de vivir el trabajo significativo y de acuerdo a los autores antes citados, se encuentra un común denominador, con frecuencia relacionan la experiencia positiva con la experiencia intrínseca.

\subsubsection{Experiencia negativa.}

En términos generales hay poca literatura con respecto a la posible experiencia negativa de vivir el trabajo significativo. Sin embargo, en el caso de Bailey \& Madden (2017), quienes han investigado el concepto de trabajo significativo en cuanto a su beneficio, recientemente encuentran una brecha de conocimiento que tiene que ver a la posible experiencia negativa que se pueda generar.

Lo anterior tiene que ver con que la experiencia del trabajo significativo va mas allá del ambiente organizacional, al trastocar la vida personal del individuo. El trabajo significativo, como argumenta Bailey \& Madden (2017); puede ser una experiencia profunda y en ocasiones conmovedora e incómoda. Puede surgir de manera inesperada, permitiendo al individuo una pausa para pensar no solo en el trabajo, sino en la vida misma, al experimentar el trabajo como significativo, dejando así de ser trabajador y reconociéndose como un ser humano.

\subsubsection{Continuidad}

Harpaz y Fu (2002), sostienen que a través de cinco construcciones se lleva al trabajo significativo a la continuidad a través del tiempo. Las construcciones o dimensiones que describen son: centralidad laboral como rol vital, normas sociales sobre el trabajo, resultados laborales valorados, importancia de los objetivos laborales e identificación del rol laboral.

La significación es un fenómeno continuo que se desarrolla día a día, no es un estado final o un objetivo al cual llegar, Ryff and Singer (1998), sino que es permanente en el tiempo.

Lo anterior vuelve atractivo el término de trabajo significativo continuo en el tiempo, dadas las condiciones actuales que no son a las que el mundo conocía como normales u ordinarias en el contexto individual y organizacional.

Como es sabido, la pandemia del COVID19, vino a modificar todo sentido y 
significancia que el individuo tenía de su vida, su dinámica social y organizacional. Los escenarios y la forma de conducirse en la sociedad son otros. Y entonces, retomamos la pregunta: ¿En tiempos de COVID-19, es relevante la significancia de trabajo?

\section{MÉTODO.}

En este estudio se consideran definiciones que por su descripción se aprecia con claridad el sentido del concepto.

Se realiza una investigación descriptiva de 21 artículos publicados en revistas científicas reconocidas de diferentes partes del mundo, principalmente Estados Unidos y Europa, por el período de 1974 a 2020. Se diseñó tabla de definiciones, donde se identifican elementos similares que lograron determinar dimensiones denominadas para este estudio experiencias: intrínseca, extrínseca, positiva, negativa y de continuidad.

Se cuantificaron resultados, con el propósito de llevarlos a porcentajes que mostraran la información pertinente del análisis y resultados.

Una limitante para este estudio fue que algunos autores no tienen una definición propia del concepto de trabajo significativo, sino que citan a otro, disminuyendo el número de consultas.

Con la información analizada se diseñó el modelo dicotómico de las definiciones de trabajo significativo, en el cual se muestra en la Figura 1 de los anexos.

\section{RESULTADOS.}

Como se observó al inicio del estudio, desde las raíces etimológicas del concepto de "trabajo significativo", se identifican aspectos dicotómicos y, aunque en menor frecuencia antagónicos, que conducen a un entendimiento distorsionado del concepto de trabajo significativo.

Se encontró dicotomía con respecto a lo intrínseco o extrínseco de la experiencia de vivir el trabajo significativo. Lo anterior quiere decir que, de manera interna y de acuerdo con creencias, cultura o valores, el individuo es capaz de vivir de forma autónoma y por voluntad propia, la experiencia del trabajo significativo. Mientras que, por otro lado, el individuo requiere de la interacción con elementos externos para lograr vivir la experiencia del trabajo significativo.

Adicional a lo anterior, también se identificaron antagonismos al identificar que el trabajo significativo se considera como positivo en cuanto al valor que se le atribuye, mientras que por otro lado puede ser considerado como negativo, dado el estrés o incomodidad que puede producir el vivirlo y llevarlo al ámbito personal.

Los porcentajes en los resultados muestran la forma en la que se presenta la dicotomía:

Gráfica 1. Porcentajes de dimensiones.



Fuente: Elaboración propia.

Como se observa en el estudio de 21 artículos, los resultados fueron que en las dimensiones dicotómicas en cuanto a la 
experiencia intrínseca hubo un $29 \%$ de frecuencia. Mientras que la experiencia extrínseca muestra un $33 \%$, con tan solo 4 puntos porcentuales de diferencia.

Por otro lado, la brecha de antagonismo es amplia. La experiencia resulta positiva en un $52 \%$, mientras que la experiencia negativa está en un $5 \%$.

Es importante señalar que algunos autores, puntualizan de dos a tres dimensiones en un estudio.

Con los resultados obtenidos se diseñó el modelo dicotómico de las definiciones de trabajo significativo, donde incluso se hace alusión al antagonismo identificado. Ver Figura 1.

Figura 1. Modelo dicotómico de las definiciones de trabajo significativo.

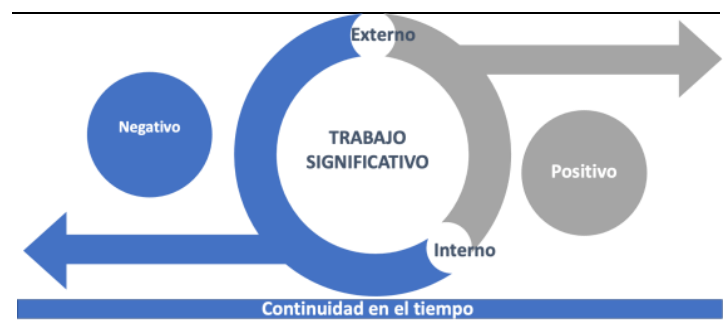

Fuente: elaboración propia.

En el Modelo dicotómico de las definiciones del trabajo significativo se aprecia como la experiencia intrínseca y extrínseca forman parte del individuo, sin embargo, existe una separación desde el interior hasta el exterior. Mientras, que se muestra cierto antagonismo, al identificar al trabajo significativo como positivo o negativo en determinadas circunstancias

\section{CONCLUSIONES.}

Con los resultados obtenidos, se concluye que, en el análisis de las definiciones propuestas por los diferentes autores, existe dicotomía y antagonismo. Lo anterior quiere decir que la percepción del concepto de trabajo significativo hasta hoy podría llevar a confusión en cuanto a su percepción.

Por otro lado, pero en el mismo sentido, se propone la identificación de atributos que lleven a una clasificación por dimensiones, en donde se especifiquen los atributos que las componen, con el propósito de contribuir a una homogeneidad en la definición del concepto.

Lo anterior dado que, con las definiciones analizadas, se observa que de acuerdo con los escenarios en dónde el individuo es el actor principal, la dinámica de la experiencia del trabajo significativo se puede mover desde una experiencia interna y particular; que tiene que ver con la emocionalidad con que el individuo le da sentido a su vida y por lo tanto le invierte energía a su trabajo (Korotkov, D.L. 1998). Hasta que la misma experiencia interna sea impactada por estímulos externos intensos, y volverse una experiencia extrínseca, además de positiva o negativa; incluso con incomodidad, llegando a convertirse en un sacrificio personal, si el empleado superpone el trabajo sobre su tiempo libre y familiar Bailey \& Madden (2017). Y entonces se asume que con un evento como la pandemia de COVID-19, el propio indivduo encuentra su trabajo significativo por el beneficio económico, pero a la vez inseguro por los contagios.

Otro hallazgo que es interesante para un estudio posterior es la posible continuidad en el tiempo de la experiencia del trabajo significativo. 


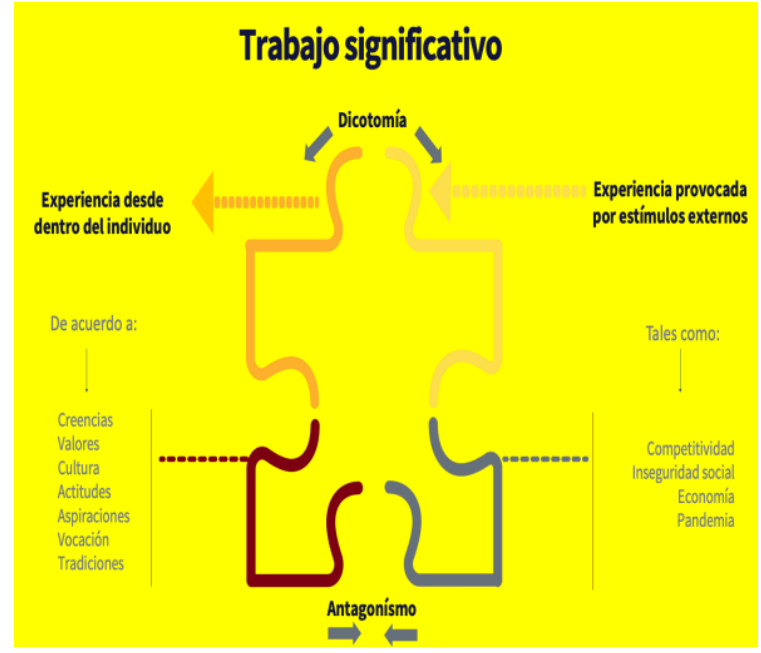




\section{REFERENCIAS}

Allan, B. A., \& Liu, T. (2020). Transitions in an uncertain labor market: implications for meaningful work. In Navigating Life Transitions for Meaning (pp. 91-104). Academic Press.d

Bailey, C., Madden, A., Alfes, K., Shantz, A., \& Soane, E. (2017). The mismanaged soul: Existential labor and the erosion of meaningful work. Human Resource Management Review, 27(3), 416-430.

Baum, S. K., \& Stewart Jr, R. B. (1990). Sources of meaning through the lifespan. Psychological Reports, 67(1), 3-14.

Both-Nwabuwe, J., Dijkstra, M., \& Beersma, B. (2017). Sweeping the floor or putting a man on the moon: How to define and measure meaningful work. Frontiers in psychology, 8, 1658.

Brief, A. P., \& Nord, W. R. (Eds.). (1990). Meanings of occupational work: A collection of essays. Free Press.

Burke, P. J., \& Stets, J. E. (2009). Identity theory. Oxford University Press.

Chalofsky, N. (2003). An emerging construct for meaningful work. Human Resource Development International, 6(1), 69-83.

Ciulla, J. B. (2005). The state of leadership ethics and the work that lies before us. BUSINESS ETHICS-OXFORD-A EUROPEAN REVIEW-, 14(4), 323.

Harpaz, I., \& Fu, X. (2002). The structure of the meaning of work: A relative stability amidst change. Human relations, 55(6), 639-667.

Korotkov, D. L. (1998). The sense of coherence: Making sense out of chaos. Lawrence Erlbaum Associates Publishers.

Lips-Wiersma, M., \& Morris, L. (2009). Discriminating between 'meaningful work'and the 'management of meaning'. Journal of business ethics, 88(3), 491-511.

Martela, F., \& Steger, M. F. (2016). The three meanings of meaning in life: Distinguishing coherence, purpose, and significance. The Journal of Positive Psychology, 11(5), 531-545.

Mitra, R., \& Buzzanell, P. M. (2017). Communicative tensions of meaningful work: The case of sustainability practitioners. Human Relations, 70(5), 594-616.

MOW International Research Team. (1987). The meaning of working. Academic Pr.

Nikolova, M., \& Cnossen, F. (2020). What makes work meaningful and why economists should care about it. Labour Economics, 101847.

Real Academia de la lengua Española

Richard, H. J., \& Oldham, G. (1976). Motivation through the design of work: Test of a theory. Organizational behavior and human performance, 16(2), 250-279. 
Romero Caraballo, M. P. (2017). Work meaning from work psychology. A historical, psychological and sociological revision. Psicología desde el Caribe, 34(2), 120-138.

Rosso, B. D., \& Wrzesniewski, A. (2010). One the Meaning of work: A theoretical integration and review. Research in organizational Behavior, 30, 91-127.

Ryff, C. D., \& Singer, B. (1998). The contours of positive human health. Psychological inquiry, 9(1), $1-28$.

Schabram, K., \& Maitlis, S. (2017). Negotiating the challenges of a calling: Emotion and enacted sensemaking in animal shelter work. Academy of Management Journal, 60(2), 584-609.

Steger, M. F., Dik, B. J., \& Duffy, R. D. (2012). Measuring meaningful work: The work and meaning inventory (WAMI). Journal of career Assessment, 20(3), 322-337.

Stephan, U., Tavares, S. M., Carvalho, H., Ramalho, J. J., Santos, S. C., \& van Veldhoven, M. (2020). Self-employment and eudaimonic well-being: Energized by meaning, enabled by societal legitimacy. Journal of Business Venturing, 35(6), 106047.

Wrzesniewski, A., \& Dutton, J. E. (2001). Crafting a job: Revisioning employees as active crafters of their work. Academy of management review, 26(2), 179-201.

Yeoman, R. (2014). Conceptualising meaningful work as a fundamental human need. Journal of Business Ethics, 125(2), 235-251.

Anexos.

Tabla 1. Tabla de definiciones de trabajo significativo con clasificación de experiencia.

\begin{tabular}{cccc}
\hline Año & Autor & Definición & Experiencia \\
\hline 1976 & Hackman \& & Grado en el que el empleado experimenta el trabajo como uno que & $\bullet$ \\
\hline
\end{tabular}




\begin{tabular}{|c|c|c|c|}
\hline & $\begin{array}{l}\text { Oldham } \\
(1976) \text {. }\end{array}$ & generalmente es significativo, valioso y que vale la pena". & \\
\hline 1987 & $\begin{array}{l}\text { MOW } \\
(1987) \\
\text { Internationa } \\
1 \text { Research } \\
\text { team }\end{array}$ & $\begin{array}{l}\text { Conjunto de creencias, valores y actitudes que se van construyendo } \\
\text { desde el núcleo familiar y que varían de acuerdo con las experiencias } \\
\text { subjetivas y los diferentes aspectos situacionales que suceden en el } \\
\text { contexto laboral y organizacional. }\end{array}$ & $\begin{array}{l}\text { - Intrínseca } \\
\text { - Extrínseca }\end{array}$ \\
\hline 1990 & $\begin{array}{l}\text { Baum \& } \\
\text { Stewart } \\
(1990)\end{array}$ & $\begin{array}{l}\text { El significado se define como dar sentido a las partes componentes de la } \\
\text { existencia de uno, mientras que el propósito implica la intención y la } \\
\text { búsqueda de objetivos: el lenguaje para ambos términos se usa a menudo } \\
\text { indistintamente y puede ser conceptualmente diferente. }\end{array}$ & - Positiva \\
\hline 1998 & $\begin{array}{l}\text { Ryff and } \\
\text { Singer } \\
(1998) \text {. }\end{array}$ & $\begin{array}{l}\text { Fenómeno continuo que se desarrolla día a día, no un estado final que se } \\
\text { resuelva de una vez por todas" }\end{array}$ & - Continuidad \\
\hline 1998 & $\begin{array}{l}\text { Korotkov, } \\
\text { D. L. } \\
(1998) \text {. }\end{array}$ & $\begin{array}{l}\text { El significado se refiere al grado en que la vida tiene sentido emocional } \\
\text { y que las demandas que enfrenta se perciben como un valor de inversión } \\
\text { y compromiso energético" }\end{array}$ & $\begin{array}{l}\text { - Intrínseca } \\
\text { - Extrínseca } \\
\text { - Positiva }\end{array}$ \\
\hline 2005 & $\begin{array}{l}\text { Ciulla } \\
(2005)\end{array}$ & $\begin{array}{l}\text { El significado es interno y subjetivo: las personas difieren en lo que } \\
\text { encuentran significativo. Sin embargo, también es externo u objetivo, ya } \\
\text { que lo que sucede en el mundo da forma a lo que hacemos y no } \\
\text { encontramos significativo; en todo tipo de actividades, ya sea que } \\
\text { forman parte de un empleo remunerado o de tiempo libre. }\end{array}$ & $\begin{array}{l}\text { - Intrínseca } \\
\text { - Extrínseca }\end{array}$ \\
\hline 2001 & $\begin{array}{l}\text { Wrzesniews } \\
\text { ki \& Dutton } \\
(2001) \text {. }\end{array}$ & $\begin{array}{l}\text { Describe tres orientaciones diferentes que pueden afectar la disposición } \\
\text { para encontrar algo significativo en el trabajo: Empleo: El trabajo como } \\
\text { una fuente de bienestar que activa otras áreas de la vida. La satisfacción } \\
\text { más grande viene de afuera, de los hobbies y de las relaciones } \\
\text { interpersonales. El significado del empleo es primordialmente lo que } \\
\text { contribuye a los ámbitos externos de la vida. Carrera: El trabajo como } \\
\text { una fuente de avance, prestigio y status. Llamado o Vocación. La gente } \\
\text { con una orientación profesional de este tipo generalmente está dispuesta } \\
\text { a hacer sacrificios (que otros no harían) por sobresalir. }\end{array}$ & $\begin{array}{l}\text { - Extrínseca } \\
\text { - Positiva }\end{array}$ \\
\hline 2002 & $\begin{array}{l}\text { Harpaz y Fu } \\
(2002) \text {. }\end{array}$ & $\begin{array}{l}\text { El trabajo lo definen a través de cinco dominios o construcciones } \\
\text { principales: centralidad laboral como rol vital, normas sociales sobre el } \\
\text { trabajo, resultados laborales valorados, importancia de los objetivos } \\
\text { laborales e identificación del rol laboral. }\end{array}$ & - Extrínseca \\
\hline 2009 & $\begin{array}{l}\text { MarjoleinLi } \\
\text { ps-Wiersma } \\
\text { Lani (2009). }\end{array}$ & $\begin{array}{l}\text { El significado existencial de la significación que tiene lugar entre el } \\
\text { "ideal" y la "realidad". Las cuatro fuentes del trabajo significativo: } \\
\text { desarrollarse y convertirse en uno mismo, unidad con los demás, servir } \\
\text { a los demás y expresarse a sí mismo, aclarando que cuando se refieren a } \\
\text { la comunicación expresarse para aproximarse más a un significado más } \\
\text { completo, que también incluía el activismo. }\end{array}$ & $\begin{array}{l}\text { - Intrínseca } \\
\text { - Extrínseca }\end{array}$ \\
\hline 2010 & $\begin{array}{l}\text { Rosso, } \\
\text { Dekas, and } \\
\text { Wrzeniewsk } \\
\text { i, }(2010) \text {. }\end{array}$ & $\begin{array}{l}\text { El trabajo significativo es un componente fundamental en el bienestar. } \\
\text { Cantidad o el grado de importancia que los empleados creen que posee } \\
\text { su trabajo. } \\
\text { "Un resultado generalmente positivo o beneficioso para las personas y } \\
\text { las organizaciones. Es un trabajo que se experimenta como } \\
\text { particularmente significativo y que tiene un significado más positivo } \\
\text { para los individuos. } \\
\text { El constructo significación tiene un valor positivo en la literatura por lo } \\
\text { que mayores cantidades de significación experimentada son más } \\
\text { positivas. Por lo tanto, el "trabajo significativo" es un trabajo que se } \\
\text { experimenta como particularmente significativo y con significado } \\
\text { positivo para las personas. }\end{array}$ & - Positiva \\
\hline
\end{tabular}

Fuente: Elaboración propia.

Tabla 2. Tabla de definiciones de trabajo significativo con clasificación de experiencia.

\begin{tabular}{llll}
\hline Año & Autor & Definición & Experiencia \\
\hline
\end{tabular}




\begin{tabular}{|c|c|c|c|}
\hline 2012 & $\begin{array}{l}\text { (Steager, } \\
\text { Dik, \& } \\
\text { Duffy, } \\
\text { 2012). }\end{array}$ & $\begin{array}{l}\text { El Trabajo Significativo consiste (en al menos) una variedad de } \\
\text { habilidades, la oportunidad de concluir una actividad completa } \\
\text { (identidad de la tarea), importancia de la tarea para otras personas, } \\
\text { orgullo militar, compromiso, un sentido de vocación, desafío, } \\
\text { identidad del rol, centralidad laboral, valores laborales, orientación } \\
\text { intrínseca al trabajo, espiritualidad, buena paga y reputación. }\end{array}$ & $\begin{array}{l}\text { - Intrínseca } \\
\text { - Extrínseca }\end{array}$ \\
\hline 2014 & $\begin{array}{l}\text { Yeoman, R. } \\
\text { (2014). }\end{array}$ & $\begin{array}{l}\text { Realizar un trabajo significativo se considera una necesidad } \\
\text { económica básica y fundamental. }\end{array}$ & - Extrínseca \\
\hline 2016 & $\begin{array}{l}\text { Bailey \& } \\
\text { Madden } \\
(2017)\end{array}$ & $\begin{array}{l}\text { El trabajo significativo es una experiencia que va más allá del lugar de } \\
\text { trabajo y llega a los ámbitos de la vida personal del individuo, puede } \\
\text { ser una experiencia muy profunda, conmovedora e incluso incómoda. } \\
\text { Surge raramente y a menudo de manera inesperada, dando al individuo } \\
\text { una pausa para pensar no solo en el trabajo, sino en la vida misma, al } \\
\text { experimentar nuestro trabajo como algo significativo, dejamos de ser } \\
\text { trabajadores o empleados y nos convertimos en seres humanos. }\end{array}$ & $\begin{array}{l}\text { - Extrínseca } \\
\text { - Negativa }\end{array}$ \\
\hline 2016 & $\begin{array}{l}\text { Martela, \& } \\
\text { Steger } \\
(2016)\end{array}$ & $\begin{array}{l}\text { La significación se trata de un sentido de valor inherente de la vida y } \\
\text { de tener una vida que valga la pena vivir. La significación en el sentido } \\
\text { más amplio se refiere a la significación del trabajo como una } \\
\text { evaluación general del trabajo en cuanto a si es intrínsecamente valioso } \\
\text { y vale la pena realizarlo. }\end{array}$ & - Positiva \\
\hline 2017 & $\begin{array}{l}\text { Romero } \\
\text { Caraballo } \\
(2017)\end{array}$ & $\begin{array}{l}\text { Es una variedad de representaciones propias de cada individuo, que } \\
\text { se tienen respecto al trabajo, a través de las cuales se establecen } \\
\text { relaciones, identidades y la noción del manejo del tiempo, en función } \\
\text { de las expectativas personales con el proceso trabajo. }\end{array}$ & - Interna \\
\hline 2017 & $\begin{array}{l}\text { Mitra \& } \\
\text { Buzzanell, } \\
(2017) \text {. }\end{array}$ & $\begin{array}{l}\text { Significación como experiencia principalmente positiva para los } \\
\text { empleados, a menudo asociada con la pasión, el equilibrio entre la vida } \\
\text { laboral y personal o la creatividad. }\end{array}$ & - Positiva \\
\hline 2017 & $\begin{array}{l}\text { Both- } \\
\text { Nwabuwe, } \\
\text { Dijkstra \& } \\
\text { Beersma } \\
\text { (2017). } \\
\end{array}$ & $\begin{array}{l}\text { La experiencia subjetiva de importancia existencial se refiere al } \\
\text { proceso de percibir personalmente el trabajo como una contribución o } \\
\text { dar un sentido de la razón de ser de uno mismo en el mundo. }\end{array}$ & - Positiva \\
\hline 2017 & $\begin{array}{l}\text { Bailey, } \\
\text { Madden, } \\
\text { Alfes, } \\
\text { Shantz \& } \\
\text { Soane } \\
(2017) \text {. }\end{array}$ & $\begin{array}{l}\text { El trabajo significativo se ha definido como un trabajo que es } \\
\text { personalmente enriquecedor y que hace una contribución positiva. }\end{array}$ & - Positiva \\
\hline 2020 & $\begin{array}{l}\text { Nikolova \& } \\
\text { Cnossen } \\
(2020) \text {. }\end{array}$ & $\begin{array}{l}\text { Actividades relacionadas con el trabajo que las personas consideran } \\
\text { útiles y valiosas. }\end{array}$ & - Positiva \\
\hline 2020 & $\begin{array}{l}\text { Allan \& Liu } \\
(2020) \text {. }\end{array}$ & $\begin{array}{l}\text { El trabajo significativo como una experiencia intrínsecamente valiosa } \\
\text { y como un factor crítico para facilitar resultados positivos en el lugar } \\
\text { de trabajo, como el compromiso laboral, la satisfacción laboral y el } \\
\text { desempeño. }\end{array}$ & $\begin{array}{ll}\text { - } & \text { Interna } \\
\text { - } & \text { Positiva }\end{array}$ \\
\hline 2020 & $\begin{array}{l}\text { Stephan, } \\
\text { Tavares, } \\
\text { Carvalho, } \\
\text { Ramalho, } \\
\text { Santos, \& } \\
\text { van } \\
\text { Veldhoven } \\
(2020) .\end{array}$ & $\begin{array}{l}\text { La significación en el trabajo es la experiencia del trabajo como } \\
\text { enriquecedor y útil personalmente. El trabajo como una forma de } \\
\text { expresarse a sí mismo y una fuente de motivación intrínseca conduce } \\
\text { a un mayor sentido de propósito y significado }\end{array}$ & - Positiva \\
\hline
\end{tabular}

